\title{
Détermination de la teneur en enzymes des coagulants animaux. Simplification de la méthode officielle de dosage.
}

\author{
AM Tanty, JL Brelle \\ Sochal, 15400 St-Etienne-de-Chomeil, France
}

(Reçu le 7 août 1991; accepté le 30 octobre 1991)

\begin{abstract}
Résumé - Le protocole officiel de détermination, par chromatographie, des teneurs en chymosine et en pepsine bovine des coagulants animaux (Norme FIL 110A:1987) a été modifié par suppression de la phase de dialyse des échantillons, réduction de la hauteur de résine dans la colonne et diminution des volumes d'éluats recueillis. L'application de cette méthodologie simplifiée permet de diviser par trois le délai de réponse de l'analyse et fournit des résultats plus fiables et plus reproductibles que ceux découlant de la méthode normalisée.
\end{abstract}

présure / chymosine / pepsine bovine / dosage / chromatographie

Summary - Determination of chymosin and bovine pepsin in rennets. Simplification of the IDF normalized method. The official method for determination of chymosin and bovine pepsin in bovine rennets and pepsins (IDF 110A:1987) has been modified in a simplified way: sample dialysis was suppressed and column height and elutions were shortened. The proposed simplified method gives reproducible and accurate results and takes 3 times less time than the normalized method.

rennet / chymosin / bovine pepsin / determination / chromatography 


\section{INTRODUCTION}

Les coagulants animaux (présures, extraits de pepsine bovine et leurs mélanges) ont tous en commun de ne contenir que 2 enzymes principales : la chymosine et la pepsine bovine. Seules changent les proportions respectives de ces 2 enzymes, dont les paramètres d'activité protéasique sont bien distincts.

Les travaux de Garnot et al (1972) complétés par Collin et al (1981), ont débouché en France sur l'établissement d'une méthode officielle d'analyse des coagulants animaux publiée au Journal Officiel de la République Française (1981). Cette méthode, récemment normalisée au plan international par la Fédération internationale de laiterie (norme 110A : 1987), est devenue aujourd'hui un standard analytique reconnu.

$\mathrm{Si}$, depuis près de 10 ans, les utilisateurs réguliers de la méthode ont pu en vérifier la fiabilité, par un suivi rigoureux du protocole expérimental, le temps nécessaire à la réalisation de l'analyse chromatographique (environ $6 \mathrm{~h}$ ) reste encore un frein à une pratique plus large ou plus systématique de cette analyse.

Nous avons cherché dans la présente étude à apporter de légères modifications au protocole normalisé, sans en remettre le principe en cause, afin de réduire les temps d'analyse et les délais d'obtention des résultats. Ce protocole simplifié a été pratiqué parallèlement à celui décrit par la FIL. La précision et la fiabilité des résultats obtenus ont été discutées.

\section{MATÉRIEL ET MÉTHODE}

\section{Matériel et réactifs}

L'appareillage et les réactifs nécessaires à l'analyse chromatographique étaient conformes aux prescriptions données par la norme FIL.
La poudre de lait étalonnée (lot $n^{\circ} 5$ ) et la solution standardisée de $\mathrm{CaCl}_{2}$ étaient fournies par I'INRA (BP94, 39800 Poligny).

Cinq échantillons liquides de coagulants animaux ont été analysés dans cette étude : un "extrait de présure $520 \mathrm{mg}$ " (EP), un «mélange $3 / 1$ " (3/1), un «mélange $2 / 2$ " (2/2), un "extrait de pepsine bovine $1800 \mathrm{mg}$ " (EPB), et un produit, appelé ci-après "30/70" intermédiaire entre le mélange $2 / 2$ et l'extrait de pepsine bovine au niveau de sa composition enzymatique.

\section{Méthode}

Chaque échantillon de coagulant a été analysé à plusieurs reprises et en parallèle avec, d'une part, la méthode normalisée FIL/IDF, et d'autre part cette même méthode modifiée sur les points suivants :

- la suspension de cellulose DEAE introduite dans les colonnes de chromatographie représentait une hauteur de 3 à $3,5 \mathrm{~cm}$ après décantation (au lieu de $9 \mathrm{~cm}$ selon le protocole FIL):

- l'échantillon à analyser n'était pas dialysé mais dilué dans $20 \mathrm{ml}$ de tampon 1 afin d'obtenir une préparation dont la molarité en $\mathrm{NaCl}$ soit de 0,2 $\mathrm{mol} / \mathrm{l}$ au plus;

- les colonnes de chromatographies étaient équilibrées avec le tampon 2;

- les volumes d'échantillon dilué déposés sur colonne calculés sur les bases des prescriptions de la méthode officielle FIL en tenant compte du facteur de dilution de chaque échantillon dans le tampon 1 étaient compris entre 2 et $4,5 \mathrm{ml}$;

- après le dépôt sur colonne, une première fraction de $25 \mathrm{ml}$ contenant la chymosine a été éluée avec le tampon 2, et une deuxième de $25 \mathrm{ml}(50 \mathrm{ml}$ pour l'échantillon EPB) contenant la pepsine bovine, a été éluée avec le tampon 3 . Le débit de la pompe péristaltique était réglé à $80 \mathrm{ml} / \mathrm{h}$. Après recueil de la première fraction, une fraction intermédiaire de $2-3 \mathrm{ml}$ était recueillie pour vérifier la complète élution de la chymosine.

Les mesures d'activité coagulante ont été effectuées selon la méthodologie décrite dans la norme FIL 110A. Les compositions relatives (taux d'activité pepsine) ont été calculées selon le protocole FIL. 


\section{RÉSULTATS \\ Détermination du taux d'activité pepsine}

La synthèse des résultats d'analyse des taux d'activité pepsine pour chaque type d'échantillons est reportée dans le tableau I. Globalement, les résultats moyens otenus par les méthodes diffèrent assez peu. Seul le mélange $3 / 1$ montre une différence assez sensible entre les résultats obtenus par les 2 méthodes.

Par ailleurs, on peut noter que la méthode simplifiée offre une reproductibilité légèrement meilleure que la méthode FIL (écarts types d'échantillonnages globalement plus faibles).

\section{Analyse statistique des résultats}

L'analyse de régression effectuée entre les 2 séries de valeurs mesurées révèle une très bonne répétabilité des mesures quelle que soit la méthode utilisée et quelle que soit la nature des produits analysés (tableau II). Le coefficient de corrélation entre les 2 séries de résultats par les 2 protocoles opératoires est supérieur à 0,999.

\section{Validité des résultats obtenus par la méthode simplifiée}

Comme l'indiquent les résultats présentés dans le tableau I, l'application de la méthode simplifiée a donné des valeurs d'activité pepsine toujours inférieures en moyenne à celles obtenues en suivant le protocole décrit dans la norme FIL.

En particulier, des différences assez importantes ont été relevées pour les mélanges $3 / 1$ et $2 / 2$.

Pour analyser ces écarts, 2 mélanges de type $3 / 1$ et $2 / 2$, ont été réalisés à partir d'un extrait de présure et d'un extrait de pepsine bovine concentré, préalablement analysés en chromatographie, par les 2 méthodes, à 4 reprises.

Tableau I. Mesures comparées, obtenus par les 2 méthodes chromatographiques, du taux d'activité pepsine des 5 coagulants testés.

Compared results in pepsin activity percentage on the 5 rennets tested by the 2 different chromatographic methods.

\begin{tabular}{|c|c|c|c|c|c|}
\hline Échantillon & $E P$ & $3 / 1$ & $2 / 2$ & $30 / 70$ & $E P B$ \\
\hline $\mathrm{Nb}$ de répétitions & 9 & 9 & 9 & 6 & 8 \\
\hline \multicolumn{6}{|l|}{ Méthode FIL } \\
\hline activité pepsine moyenne (\%) & 35,47 & 55,52 & 69,81 & 81,12 & 93,43 \\
\hline écart type d'échantillonnage & 0,341 & 0,284 & 0,207 & 0,230 & 0,100 \\
\hline coefficient de variation & $0,96 \%$ & $0,51 \%$ & $0,30 \%$ & $0,28 \%$ & $0,11 \%$ \\
\hline \multicolumn{6}{|l|}{ Méthode modifiée } \\
\hline activité pepsine moyenne (\%) & 35,27 & 54,26 & 69,31 & 80,91 & 93,35 \\
\hline écart type d'échantillonnage & 0,268 & 0,212 & 0,224 & 0,118 & 0,093 \\
\hline coefficient de variation & $0,76 \%$ & $0,39 \%$ & $0,32 \%$ & $0,15 \%$ & $0,10 \%$ \\
\hline
\end{tabular}


Tableau II. Étude de régression sur les 2 séries de résultats obtenus par chacune des 2 méthodes.

Regression analysis of data from both IDF and modified methods.

\section{Variable explicative Variable expliquée}

Nombre de couples de données

\section{Coefficient de régression}

Terme constant de la régression

Ecart type de la régression

Ecart type résiduel

Coefficient de corrélation $\mathrm{R}^{2}$
Résultats

méthode FIL méthode simplifiée

41
1,0063

$-0,8921$

0,0038

0,500

0,9994
Chacun des 2 mélanges a été analysé selon les 2 méthodes, 4 fois, et les moyennes des résultats ont été comparées aux résultats théoriques, calculés à partir des analyses des ingrédients de départ et des proportions des mélanges.

La formule de calcul des concentrations en enzymes que nous avons appliquée était la suivante :

$$
C=\frac{K}{T-a} \times \frac{V}{V e} \times F
$$

$C$ : teneur en $\mathrm{mg} / \mathrm{l}$ d'enzyme (chymosine ou pepsine); $K$ : facteur dépendant du lait en poudre utilisé et de l'enzyme considérée (donné par le fournisseur de poudre de lait); $T$ : temps de coagulation (s) pour la fraction considérée; a : constante dépendant du lait en poudre utilisé et de l'enzyme considérée (donnée par le fournisseur de poudre de lait); $V$ : volume de la fraction collectée (en $\mathrm{ml}$ ); $V_{e}$ : volume (en $\mathrm{ml})$ de l'échantillon déposé sur la colonne (considérer le volume d'échantillon non dilué); F : facteur de dilution de la fraction considérée.
On constate que l'application de la méthode simplifiée donne des valeurs qui sont beaucoup plus proches des valeurs théoriques de composition des mélanges que celles obtenues en suivant le protocole de la FIL. De ce fait, on peut considérer que la méthode simplifiée donne des résultats plus fiables que ceux obtenus par la méthode FIL sous sa forme actuelle.

\section{DISCUSSION - CONCLUSIONS}

Les modifications que nous avons apportées à la méthode officielle de dosage ont permis de répondre aux objectifs fixés : obtenir des résultats fiables, rapidement, selon un mode opératoire simplifié.

Le gain de temps s'exprime à la fois en gain de temps de manipulation et en raccourcissement du délai d'obtention des résultats. La suppression de la phase initiale de dialyse contribue en particulier grandement à ce gain de temps ( 2 h 30 minimum), de même que l'élution de volumes moindres (20 min par fraction au lieu de $1 \mathrm{~h} 15)$.

La seule opération supplémentaire, nécessaire à la mise en œuvre de la méthode simplifiée, est une dilution de l'échantillon à analyser dans le tampon pipérazine 1. La teneur en $\mathrm{NaCl}$ des coagulants commerciaux n'excédant pratiquement jamais $230 \mathrm{~g} / \mathrm{l}$, on peut réaliser a priori une dilution systématique à $5 \%$ afin d'obtenir une solution inférieure à $0,2 \mathrm{~mol} / \mathrm{I}$ de molarité en $\mathrm{NaCl}$.

Globalement, l'application du protocole simplifié proposé dans cette étude permet d'obtenir un résultat dans un délai plus court d'environ $4 \mathrm{~h}$ par rapport au protocole FIL.

La meilleure fiabilité des résultats issus de la méthode simplifiée découle de plusieurs points : 
- la suppression de la détermination des temps de coagulation avant et après dialyse entraîne la disparition d'une source d'erreur potentielle pour la détermination des concentrations enzymatiques;

- les volumes déposés sur la colonne étant plus importants, on peut considérer que l'erreur relative s'y rapportant est plus faible;

- le fait de pouvoir analyser les fractions issues de la séparation rapidement permet de diminuer les risques d'erreur liés à l'instabilité, d'une part de ces fractions ellesmêmes et d'autre part, du lait reconstitué. Ceci pourrait d'ailleurs expliquer pourquoi les taux d'activité chymosine ont tendance à être systématiquement plus élevés quand on utilise la méthode modifiée.

Pour être tout à fait complet, on peut souligner en outre que l'application de cette méthode simplifiée réduit les coûts matériels de l'analyse (boyau de dialyse, volumes de tampons, volumes de résine DEAE "consommée").

Tout en gardant les principes fondamentaux de la méthodologie officielle, reconnue par la législation française et normalisée par la FIL, nous avons développé une variante qui, tout en garantissant une précision et une fiabilité au moins égales voire supérieures à celles communément reconnues aujourd'hui à la méthode FIL, permet aux utilisateurs réguliers que sont les professionnels de la présure et les laboratoires de contrôles d'alléger considérablement leur charge de travail.

Les simplifications proposées ici pourront également inciter les fromagers utilisateurs de coagulants animaux, à pratiquer des autocontrôles qu'ils renonçaient à effectuer jusqu'alors devant la lourdeur apparente du protocole analytique existant.

\section{RÉFÉRENCES}

Collin JC, Martin P, Garnot P, Ribadeau Dumas B, Mocquot G (1981) Determination of chymosin and bovine pepsin A in commercial bovine rennets and pepsins. Milchwissenschaft $36,32-35$

FIL (1987) Présure de veau et pepsine bovine. Détermination des teneurs en chymosine et en pepsine bovine. Norme internationale $110 \mathrm{~A}$. Fédération Internationale de Laiterie, Square Vergote 41, 1040 Bruxelles

Garnot P, Thapon JL, Mathieu CM, Maubois JL, Ribadeau Dumas B (1972) Determination of rennin and bovine pepsins in commercial rennets and abomasal juices. J Dairy Sci 55 , $1641-1650$

Journal Officiel de la République Française, 20 mars 1981, NC 2905 